\title{
AUTHOR'S CORRECTION
}

\section{Adaptive and Mutational Resistance: Role of Porins and Efflux Pumps in Drug Resistance}

\section{Lucía Fernández and Robert E. W. Hancock}

Centre for Microbial Diseases and Immunity Research, Department of Microbiology and Immunology, University of British Columbia, Vancouver, British Columbia, Canada

Volume 25, no. 4, p. 661-681, 2012. Page 665: In Table 3, the protein AdeT is listed as a regulator of the efflux pump AdeABC, although it actually is an additional efflux protein. The first row of the table should be amended and a new row below it should be added as follows:

TABLE 3 Examples of RND efflux systems involved in the antibiotic resistance of different pathogens

\begin{tabular}{lllll}
\hline Species & Pump & Regulator(s) & Antibiotic resistance to ${ }^{a}:$ \\
\hline A. baumannii & AdeABC & AdeRS & AG, BL, CM, EM, TC, FQ & References(s) \\
A. baumannii & AdeT & & CM, EM, MI, NA, NB, TC, TM & 234 \\
\hline
\end{tabular}

Page 680: The following reference was omitted.

233a. Srinivasan VB, Rajamohan G, Pancholi P, Marcon M, Gebreyes WA. 2011. Molecular cloning and functional characterization of two novel membrane fusion proteins in conferring antimicrobial resistance in Acinetobacter baumannii. J. Antimicrob. Chemother. 66: 499-504. 